\title{
A Study of WLAN Campus in an Educational Establishment
}

\author{
doi:10.3991/ijim.v3i4.866 \\ T. Abdul-Hameed, Z. Hunaiti and E. Sedoyeka \\ Anglia Ruskin University, Chelmsford, England
}

\begin{abstract}
- the main aim of campus network is to efficiently separate, share and access knowledge among its users. The big demand of such systems is to be able to handle advanced applications which are the end-user requirements. The main goal of this study is to assess the ability of Wireless Local Area Network (WLAN) campus in dealing with applications' requests of end users in education establishments and network achievement under different conditions of operation. The study has been conducted in two stages: the first stage was accomplished by conducting a survey on the Student Village network at Anglia Ruskin University as a case study; the main aim was to determine end-use requirements, to gather information on the nature of application's running by users and to get suggestions on the likely future applications. The second stage was achieved by conducting experiments to evaluate the WLAN campus network performance under various different scenarios on the impact of handover from Access Point (AP) to another AP on end-user link performance, network performance in different usage time and network performance in different weather conditions.
\end{abstract}

Index Terms-RTT, VoIP, VPN, WLAN.

\section{INTRODUCTION}

In the last decade Internet and Information Technology have become central part at the heart of modern life and industries. Education is one of those industries, which have become dependent on the use of Information and Communication Technology (ICT) to achieve its main goals. Consequently, both teachers and students need to be fully connected through the internet in order to be able to be part of their educational process. Educational establishments are competing to build reinforcing networking infrastructures in their campuses and using remote accessing means, like Virtual Privet Network (VPN) to allow both students and staff to remain connected with their institution even if they are outside the physical boundaries of their campus. This has contributed to facilitate education allowing learners to benefit from accessing educational materials and obtained knowledge and qualifications without the need to travel using electronic learning systems. This also helped to deliver education in a wider scale without the need for building class rooms. These developments have entered into a new phase with the recent advancement in wireless communication technology where academic institutions started deploying wireless networks as a replacement or to expand their wired networks infrastructure; enabling students to benefit from accessibility to facilities everywhere across the whole campus. However, this development has been subject to the performance of wireless technology which might be fluctuating in some situations, such as changes in the weather condition, the number of the users accessing the network simultaneously and the nature of the applications running over the network. Therefore, this study has been conducted to evaluate the capabilities of WLAN campus network in handling applications demands of end users in educational establishments and network performance under different conditions of usage.

\section{BACKGROUND OF WLAN}

Wireless Local Area Networks (WLAN) is the replacement and complement to wired technology which allows computer to connect with each other using radio signal without the need of wires. WLAN, based on 802.11 b technology (commercially known as Wireless Fidelity (WiFi)), have become very common and widespread in the last few years. WiFi is a flexible data communication system technology used as an alternative to a wired LAN within a building or campus [1].

The past decade has witnessed significant changes in the type of communication services supplied to users, as well as in the infrastructure used to support them. These services, for example, can be applications with remote servers, video on demand, interactive multimedia, presentday telephony and Internet access (which occupies the biggest market). Basically, there is an overall demand for connectivity, mobility and performance. Wire line services can provide connectivity and performance, but not mobility together with connectivity. Wireless communications are the solution to these demands [2]. As a result, and in parallel with the growth of the internet, there has been significant growth in the field of wireless communications.

Wireless LANs provide connectivity, lower mobility and much higher performance in terms of achievable data transfer rate; they operate using either radio or infrared techniques. Fig. 1 gives an example of a WLAN interfacing with a wired network; they are composed of mobile units, and a wireless bridge referred to as an access point (AP). The AP interfaces the wireless network with the wired network.

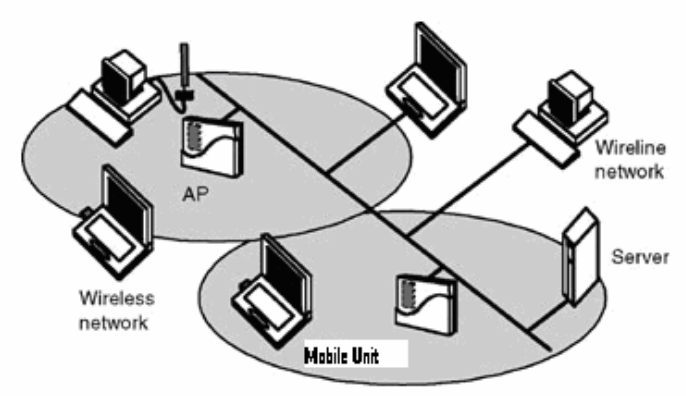

Figure 1. An example of a wireless local area network 
The most widely used WLAN (Wi-Fi) application utilises radio waves at a frequency band of $2.4 \mathrm{GHZ}$, which is also known as the Industrial, Scientific, and Medical (ISM) band. The advantage of radio waves is that they can supply connectivity for non-line-of-sight situations and it is license free, while the disadvantages are [2]:

- The electromagnetic broadcast may cause interference with equipment working in the same frequency;

- Security may be a problem, because radio waves propagate through walls.

WLAN has quickly become greatly popular. Defined by IEEE 802.11 series of standards, WLAN has quickly become popular as more users avoid the expenses and delays that come with wired networks [3].

The original 802.11 standard is the fundament of WLAN products evolved in 1997 by the Institute of Electrical and Electronics Engineers (IEEE). That base standard continues to be enhanced through documents additions such as $802.11 \mathrm{~b}, 802.11 \mathrm{a}$, or $802.11 \mathrm{~g}$. These enhancements get developments in data rate and functionality guiding to fast progression of the WLAN market [4];

- IEEE 802.11 b is a technical specification issued by the Institute of Electrical and Electronic Engineers (IEEE) that defines the operation of $2.4 \mathrm{GHz}$, $11 \mathrm{Mbps}$, Direct Spread Spectrum Wireless Local Area Networks (WLAN).

- IEEE 802.11a standard was designed for higher bandwidth applications than $802.11 \mathrm{~b}$ and includes data rates of $6,9,12.18,24,36,48,54 \mathrm{Mbps}$.

- IEEE $802.11 \mathrm{~g}$ standard was designed for data rates faster than $20 \mathrm{Mbps}$.

- IEEE $802.11 \mathrm{n}$ is a proposed amendment that improves the previous 802.11 standards.

\section{WLAN AS CAMPUS NETWORK}

The demand for mobility on campus by students and staff is increasing. A momentous number of education Institutes are having comprehensive mix of cabled Ethernet connections and they are increasingly proposing on-campus wireless services using WLAN / WiFi (802.11) technology with access to both internal applications and services and to the Internet [5].

(WLANs) are becoming increasingly popular, particularly on University and corporate campus. Previous Research [6], [7] found that nearly all plan to install a wireless network, about half already have a limited deployment, and few of them (7\%) have a "comprehensive" deployment. WLAN (Wi-Fi) will change the teaching and learning methods from passive to interactive, from oneway communication to collaboration. This feature has been installed and deployed in many Universities over the world [8].

The demand of this feature is to be capable to handle the future desire of user applications. Previous research [9], [10] found that "In fact a recent survey (2007) revealed that as high as $10 \%$ of all Internet traffic is established from just a single extremely popular video website alone". This attitude shows that we should anticipate more users in the next few years to rely on their WLAN for video applications.

Voice over IP (VoIP) calls is another WLAN application gaining popularity [9]. VoIP is a technology that al- lows telephone calls to be made over computer networks like the Internet. It converts analog voice signals into digital data packets and support real-time, two-way transmission of conversations using Internet protocol (IP) [11].

\section{CASE STUdy}

Wireless Broadband Network in the Student Village at Anglia Ruskin University's (WLAN) has been used as a site for the case study in this research project. In a personal interview with the Network Administrator at the IT Department in Anglia Ruskin University, the administrator pointed out that the network consisted of 40 Netgear abg Access points, distributed around the campus. There are between 250 and 370 students connected throughout the day, with 370 users connected at peak times (rush hour) between $1600 \mathrm{hrs}$ and $2300 \mathrm{hrs}$. The maximum theoretical speed of the network is $54 \mathrm{Mbps}$. The backbone of the network is a gigabit fibre link to the data centre, and this connects to a Bluesocket Controller, which in turn connects to a Cisco 6509, all at gigabit speed. The WAN link via JaNet is 10 gigabit, as shown in Fig. 2. To accomplish this study, two types of research methods have been used, survey and experiments.

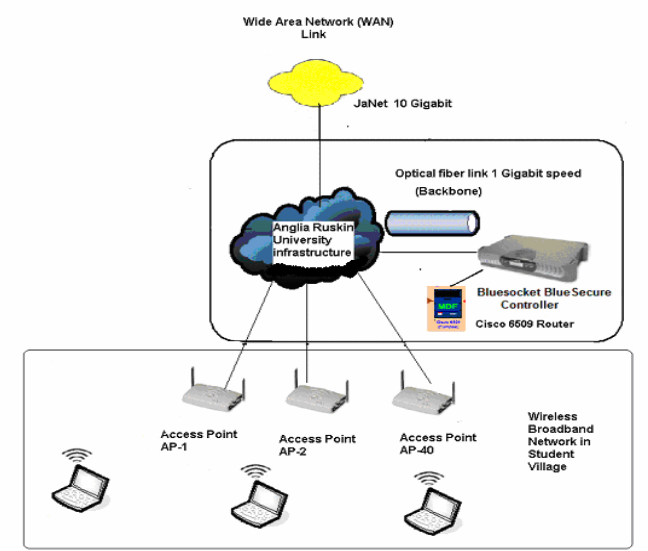

Figure 2. Anglia Ruskin University Network Architecture.

\section{Methodology}

A. Survey

A Questionnaires targeting students was distributed to 120 students and was returned by 91 of them ( 47 female and 44 male) in the case study. The response rate was $75 \%$ of the total number of users. The questionnaires were designed to achieve the following objectives:

- Evaluate and determine end-users satisfactions.

- Obtain good information on the nature applications running by users.

- Explore students' opinion on the likely future applications, like VoIP and video applications.

\section{B. Experiments}

Experiments were conducted to evaluate the WLAN campus network performance under various different scenarios:

- Network performance in different usage time. The tool that was chosen for this research was the RTT test. This test provides reports for delay, packet loss, download, Upload bandwidth speeds and other key performance measures. These measurements were 
made during different times of the day, morning, midday and evening, to cover different usage time.

- Impact of handover from Access Point (AP) to another AP on the end-user link performance. The measurement environment was the AP and a Fujitsu Siemens Laptop as a mobile station. Distances of up to $60 \mathrm{~m}$ were measured.

- Network performance in different weather conditions. Taking weather conditions into account, two different conditions were chosen when the experiments were conducted, good weather and rainy or windy weather.

RTT test was conducted by using command Ping (Packet Internet Groper). Ping is a utility associated with UNIX, the Internet, and TCP/IP networks. This method of measurement was chosen because of its simplicity and the availability of the Ping tool on all machines. It does, however, have certain limitations. Ping uses ICMP (Internet Control Message Protocol); ICMP packets can be given lower priority on some routers, or they can be blocked by firewalls. Despite these limitations, Ping data still provides good insight into the performance of a network. Each Ping received by a source host contains a value for the round trip delay between that host and the destination host. If a Ping is not returned to the source host within a specified timeout period, then it is assumed the packet has been "lost" either on the outbound or return path. The percentage of packets lost and the round trip delay are important indicators of how well certain applications will perform. By retaining data on all the Pings we are able to calculate a variety of statistics; for example, mean, medium, minimum, and maximum values. These calculations can be performed over any aggregated set of data.

\section{SURVEY RESULT}

From the data collected from the questionnaires that were distributed in the Student Village at Anglia Ruskin University, the analysis showed that:

- The survey showed that most of the students consider the quality of the Wireless Internet connection to be average as shown in Fig 3.

- On the question of what are the applications being used by students; the result was that most of them run Email activity as shown in Fig. 4.

- The survey showed that most of the students think that making phone calls over the internet is either very important or important as shown in Fig. 5. Telephone calls made over an Internet connection are predicted to be the next revolution in telecommunication, because of the cheaper broadband costs due to the number of competing companies offering these products.

- When the students were asked how important it is to be able to conduct video chat, the survey showed that most of them see it this as fairly important, as shown in Fig. 6.

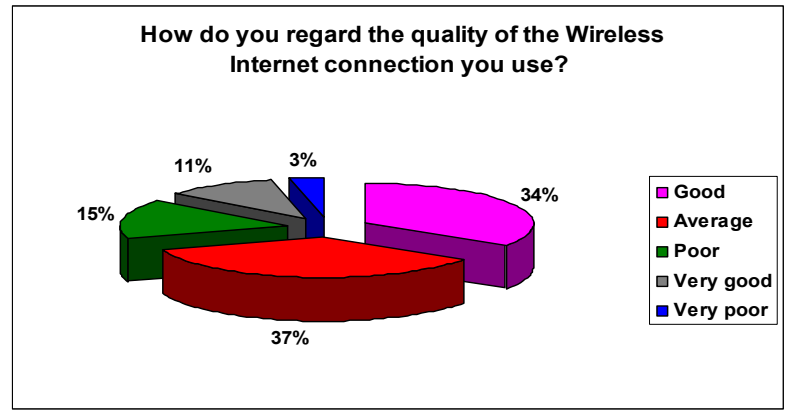

Figure 3. Student perception about the Wireless Internet connection

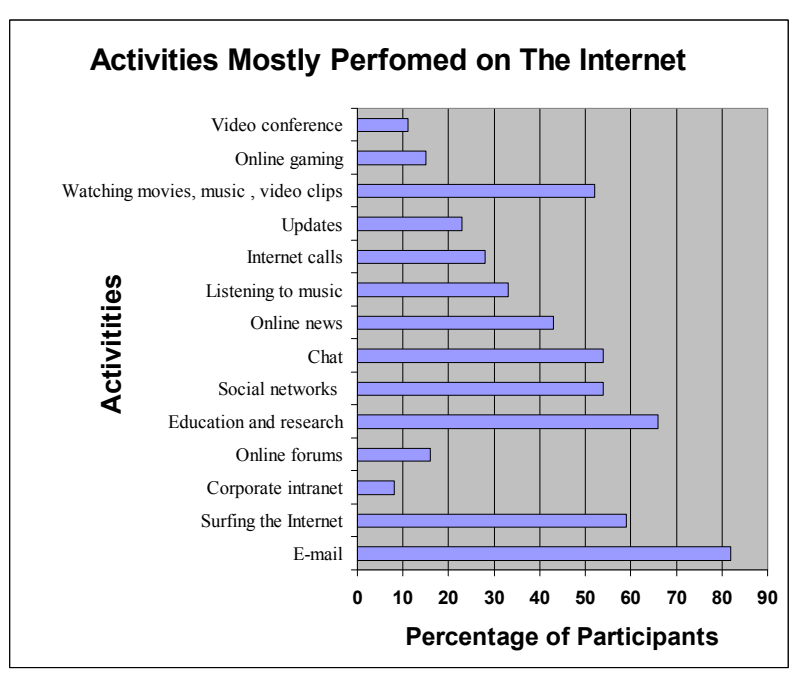

Figure 4. Nature applications running by users

How important to you to be able to make Internet calls?

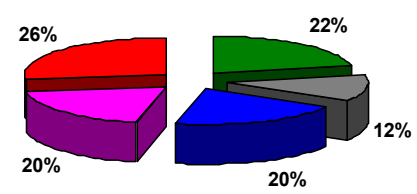

口 Less important $\square$ Slightly important - Fairly important a Important $\square$ Very important

Figure 5. Requirements of voice applications

How important to you to be able to conduct video conferences?

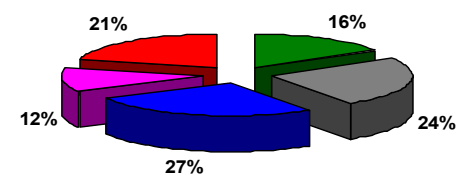

- Less important $\square$ Slightly importan - Fairly important $\square$ Important $\square$ Very important

Figure 6. Requirements of live video applications 


\section{EXPERIMENT RESULTS}

\section{A. Differnet Period Time}

Results measurement in different period time:

Latency: refers to the amount of time it takes a bit to transmit from source to destination. It is usually measured in millisecond (ms) and is one of the most important factors to be considered when developing wireless real time applications. Round Trip Time (RTT) shows the delay that the message takes to get to the destination receiver and return back to the sender; during the latency experiment the RTT test was used. The RTT test used the command Ping, by using a Fujitsu Siemens Laptop from the command line in a Disk Operating System (DOS) window under Windows XP. The results were the measurement of the Ping test from a laptop to the www.rightclicktz.com site.

The results in Fig. 7 show the average round trip latency, which is achieved at midday when the number of students who are using the network is limited.

Fig. 8 shows the results when Ping was used while downloading a file size of $9.53 \mathrm{MB}$ from the server ftp://rightclicktz.com to the laptop in different time periods. We can see that the average round trip latency is 80 $\mathrm{ms}$, when measured at midday when the number of students is less and when there was no congestion.

Fig. 9 shows the results when Ping is used while uploading a file size $9.53 \mathrm{MB}$ from the laptop to the server in different time periods. As we see in Fig 9, the average round trip also occurred around midday when the number of students decreased and there was no congestion.

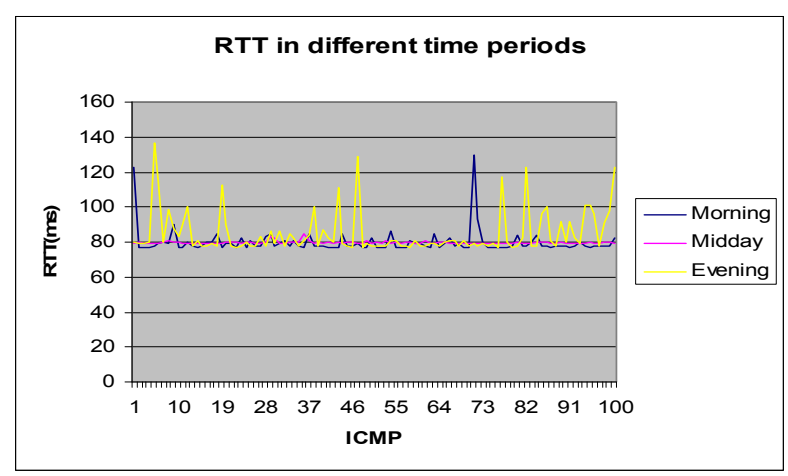

Figure 7. Round trip latency for different time periods

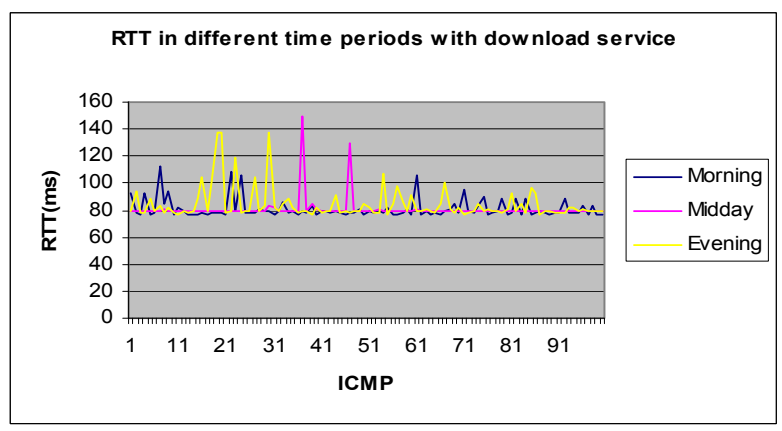

Figure 8. Round trip latency

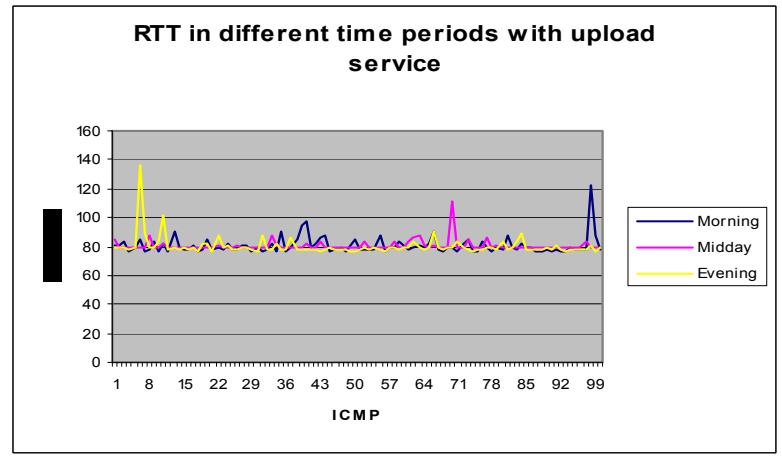

Figure 9. Round trip latency.

Bandwidth: refers to the transmission speed or throughput of our connection to the internet network. We cannot expect to see full nominal speed of our connection for the bandwidth measurement as there are always delays somewhere. In this experiment, the round trip time between the laptop and the 'ftp://rightclicktz.com' server with a file $9.53 \mathrm{MB}$ in size being downloaded or uploaded is assessed to measure the upload and download speed between the laptop and the server.

The bandwidth and transfer speed are quoted in two different units: kilobits per second (kbps) and kilobytes per second $(\mathrm{KB} / \mathrm{s})$. The difference between the two units is the number of bits in a byte, which is 8 . So, for this experiment, the file size $9.53 \mathrm{MB}$ is used, to transfer it to $\mathrm{kb}$ : $9.53 \mathrm{MB} * 8=76.24 \mathrm{Mb}^{*} 1024=78069.76 \mathrm{~kb}$. To achieve download/upload speed in $\mathrm{kbps}$, the size of the file in $\mathrm{kb}$ should be divided by the time it takes to transfer in second.

Fig. 10 shows that the download speed varies according to the download time that it takes to transfer the file from the server to the laptop, and also according to the different time periods. For example, the bandwidth measurement at midday (as shown) is much better than the bandwidth measurement in the morning and evening because the download time at midday has the lowest number of users.

Fig. 11 shows that the upload speed varies according to the upload time that it takes to transfer the file from the laptop to the server, and also according to the different time periods.

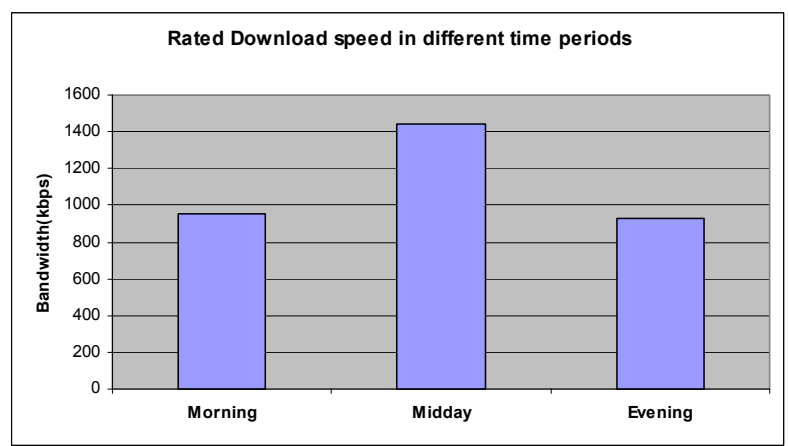

Figure 10. Throughput / Speed 


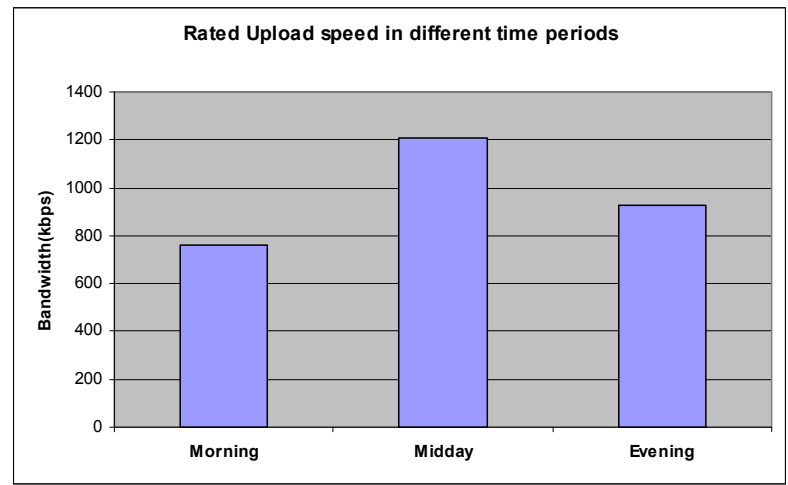

Figure 11. Throughput / Speed

Packet loss: occurs when one or more packets of data traveling across a network fail to reach their destination. It can be caused by a number of factors, including high disturbances, fading, and interference congestion. During the performance tests it was observed that there is no packet loss during morning, midday and evening hours.

\section{B. Impact of Distance from AP}

Results measurement of impact of handover from AP to another AP: RTT test method of measuring was chosen to evaluate the impact of distances from AP on end-user link performance such as Latency, Speed and packet loss. The experiment has been conducted as we were moving from the AP towards an area covered by other APs with the strongest signal strength. After choosing an AP, the mobile terminal associates to that AP and after finishing the re-association of the mobile terminal, the new AP informs the old AP of the re-association then the handover procedure is completed. We have analyzed the performance of this scenario as good and smooth because the quality remains the same as there is a handover to another AP from low quality to high quality [9].

Fig 12 shows that the latency is smooth for different distances from the AP when there is a handover to another AP.

As we see in Fig 13 the speed is also smooth and constant for different distances from AP when there is a handover between APs. It was stable at 635.34kbps.

\section{Different weather conditions}

Results of the measurement in different weather conditions showed that the delay might occur according to the network load, interference and the weather. Latency increased as the number of users increased, if there was interference, or when the weather was rainy and windy as shown in Fig. 14.

Fig. 14 shows that there is a significant difference between the results in good weather and when it is rainy or windy. The average round trip time in good weather was much less than the average RTT in rainy and windy weather.

As shown in Fig.15, there is packet lost only in bad weather. This occurs when we Ping 'rightclicktz.com' in rainy and windy weather. During the performance tests it was observed that packet loss happened only in rainy and windy weather, which is directly caused by the challenges of radio signal propagation [12], and this did not occur otherwise.

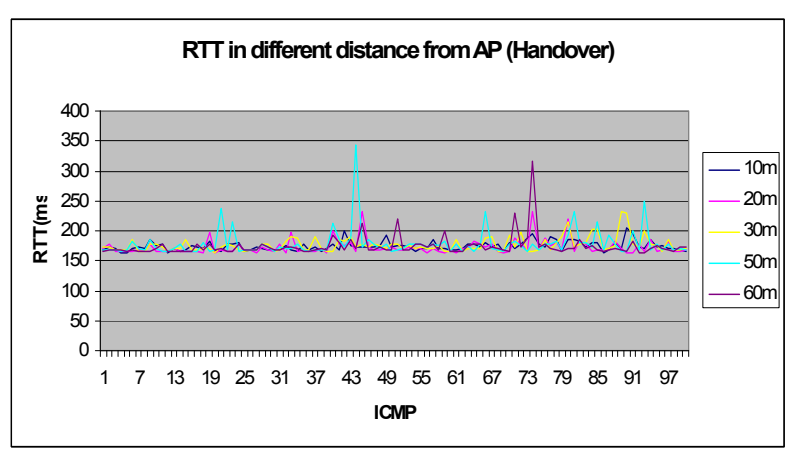

Figure 12. Round trip latency in different distance when there is a handover

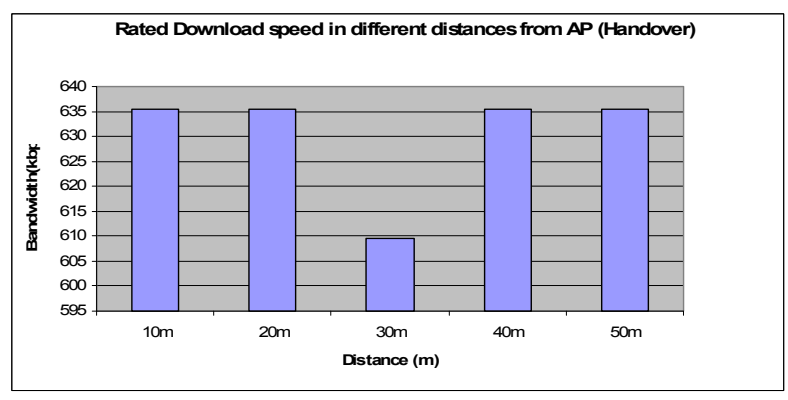

Figure 13. Throughput/Speed

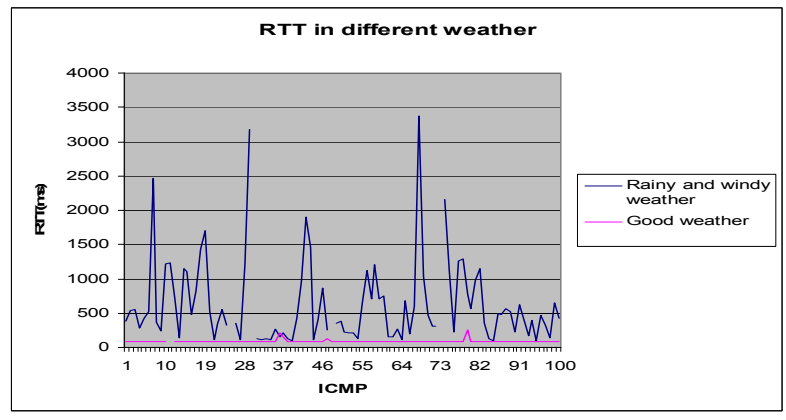

Figure 14. RTT in different weather conditions

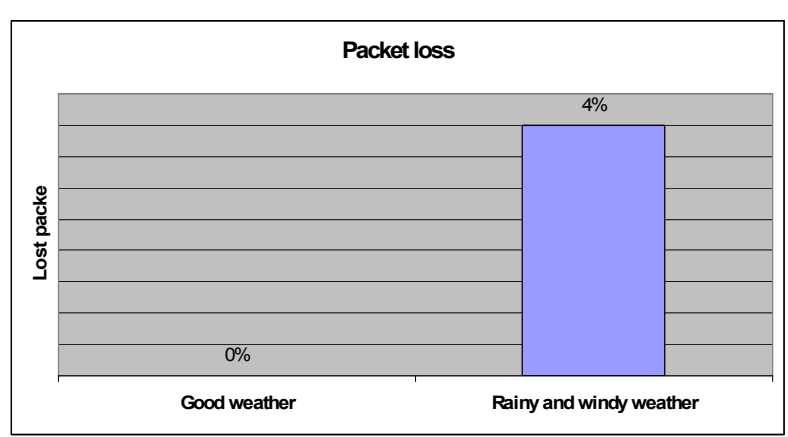

Figure 15. Packet loss for good weather vs. bad weather

\section{DISCUSSION}

The results from this study showed that the vast majority of the student participants considered the quality of Wireless Internet connection to be very good, good or average, which leads us to the conclusion that the overall student perception is that the quality of the connection (WLAN) is good. Consequently, the Student Village Wireless Network can be considered satisfactory for the students. Also, it can be seen from the results that the stu- 
dents have advanced applications requirements, as $78 \%$ of them considered making internet calls as important, $81 \%$ of them had an interest in video streaming, and $84 \%$ were interested in video conferencing or video chat. The students considered the network to be good because it is capable of running advanced internet applications according to user's requirements.

It can be concluded that the off peak time for the network is during the midday period when most of the students are at the University and there is no congestion. Also, there was no significant difference on the speed, delay and packet loss in different usage time but there was a significant difference in different weather conditions.

\section{CONCLUSION AND FURTHER WORK}

It can be concluded that WLAN Campus can offer good benefit to education including mobility, extending and conductivity across campus. That can be achieved with good planning of AP placement across the campus in order to handle changes and traffics in different time of usages (rush hours or peak time). However, WLAN network campus could suffer from dramatic drop in performance in bad weather conditions. In countries like the UK therefore, it is recommended that education establishments in countries with long winter should install WLAN as a complementary network to wired network in order to minimize this limitation. In addition, educational establishments should be aware of future demand on rich applications like VoIP and video which might be challenging for WLAN to handle in wider scale and might have direct impact on user satisfaction.

\section{REFERENCES}

[1] R. Camilo, P. Mari, M. Velasco, and J. Fuertes, "Wireless Network Delay Estimation for time-sensitive Applications", Research report ESAIIRR-06-12, p.1, Jul. 2006.

[2] R. Prasad and L. Deneire. From WPAN to Personal Networks: Technologies and Applications. Artech House, 2006. [E-book] Available: IET e-book.

[3] S. Salgar, "Emerging Technologies", International CALIBER, New Delhi, February 2004. [Online]. Available: http://dspace.inflibnet.ac.in/bitstream/1944/354/1/04cali_46.pdf [Access Jul. 23, 2008].
[4] "The New Mainstream Wireless LAN Standard", WHITE PAPER. IEEE 802.11g, Oct. 3, 2003. [Online]. Available: http://www.54g.org/pdf/802.11g-WP104-RDS1.pdf [Access Jul. 23, 2008].

[5] HEAnet:Ireland's National Education \& Research Network, "Wireless Strategy for HEAnet: Wireless Broadband Access Services", $2008 . \quad$ Available: http://www.heanet.ie/docs/20080605\%20Heanet $\% 20$ Wireless $\% 20$ Strategy final-released.pdf [Access Jul. 23, 2008].

[6] K. Essien and D. Kotz, Analysis of a campus-wide Wireless Network: Eighth Annual International Conference on mobile computing and networking, September 23-26, 2002, Atlanta, Georgia, USA.

[7] R. Boggs and P. Arabasz. The move to wireless networking in higher education. Research Bulletin of the EDUCAUSE Centre for Applied Research, April 2002.

[8] N. Graychase, "Big WLAN on Campus", Wi-Fi Planet Marketplace, Sep. 16, 2008. Available: http://www.wifiplanet.com/news/article.php/3710891[Access Jul. 24, 2008].

[9] D. Chan, "Technologies Delivering Multiuser Transmission for Next-Generation IEEE802.11 Wireless Network", Nov. 5, 2007. Available: http://www.douglaschan.com/pubs/DSChan VHT SG CSMAMPR Whitepaper.pdf [Access 24 July 2008].

[10] Ellacoya Networks Inc., "Ellacoya Data Shows Web Traffic Overtakes Peer-to-Peer (P2P) as Largest Percentage of Bandwidth on the Network," Press release, 2006.

[11] M. Bradley, "Wireless/Networking, VoIP”, Jan 5, 2009. Available: http://compnetworking.about.com/?once=true\& [Access 11 April 2009].

[12] A.S. Adegoke, "Mitigation of Effects of the Atmosphere on Radio Wave Propagation," The Pacific Journal of Science and Technology, vol.9, no. 1, May-Jun. 2008.

\section{AUTHORS}

Taghareed Abdul Hameed (tagreedhamid@aol.com)is a postgraduate student based in Anglia Ruskin University Chelmsford in department of design and technology.

Ziad Hunaiti (Ziad.Hunaiti@anglia.ac.uk)is a Lecturer at Anglia Ruskin University Chelmsford in department of design and Technology.

Eliamani Sedoyeka (amani@rightclick.com) is a Research student based in Anglia Ruskin University Chelmsford in department of design and technology.

Submitted, December, 9, 2008. Published as resubmitted by the author(s) on June, 30, 2009. 